\title{
Reducing Overuse of Proton Pump Inhibitors for Stress Ulcer Prophylaxis and Nonvariceal Gastrointestinal Bleeding in the Hospital: A Narrative Review and Implementation Guide
}

\begin{abstract}
Karen Clarke, MD ${ }^{\star *}$, Nicole Adler, MD², Deepak Agrawal, MD³, Dimpal Bhakta, MD4, Suchita Shah Sata, MD5, Sarguni Singh, MD, Arjun Gupta, MD , Amit Pahwa, MD, Emily Pherson, PharmD ${ }^{9}$, Alexander Sun, MD5, Frank Volpicelli, MD², Aditya Sreenivasan, MDº, Hyung J Cho, MD ${ }^{11,12}$

${ }^{1}$ Division of Hospital Medicine, Emory University School of Medicine, Atlanta, Georgia; ${ }^{2}$ Department of Medicine, New York University Grossman School of Medicine, New York, New York; ${ }^{3}$ Division of Gastroenterology and Hepatology, Dell Seton Medical Center, Austin, TX; ${ }^{4}$ Division of Gastroenterology, New York University School of Medicine, New York, New York; ${ }^{5}$ Department of Medicine, Duke University School of Medicine, Durham, North Carolina; ${ }^{6}$ Division of Hospital Medicine, University of Colorado, Aurora, Colorado; ${ }^{7}$ Sidney Kimmel Comprehensive Cancer Center, Johns Hopkins University School of Medicine, Baltimore, Maryland; ${ }^{8}$ Division of General Internal Medicine, Division of General Pediatrics, Johns Hopkins University School of Medicine, Baltimore, Maryland; ${ }^{9}$ Department of Pharmacy, Johns Hopkins Hospital, Baltimore, Maryland; ${ }^{10}$ Division of Gastroenterology, Long Island Jewish Medical Center, Manhasset, New York; ${ }^{11}$ Division of Hospital Medicine, Icahn School of Medicine at Mount Sinai, New York, New York; ${ }^{12 N e w ~ Y o r k ~ C i t y ~ H e a l t h ~ a n d ~ H o s p i t a l s, ~ N e w ~ Y o r k, ~ N e w ~ Y o r k . ~}$
\end{abstract}

Proton pump inhibitors (PPIs) are among the most commonly used medications in the world; however, these drugs carry the risk of patient harm, including acute and chronic kidney disease, Clostridium difficile infection, hypomagnesemia, and fractures. In the hospital setting, PPIs are overused for stress ulcer prophylaxis and gastrointestinal bleeding, and PPI use often continues after discharge. Numerous multifaceted interventions have demonstrated safe and effective reduction of PPI use in the inpatient setting. This narrative review and the resulting implementation guide summarize published interventions to reduce inappropriate PPI use and provide a strategy for quality improvement teams. Journal of Hospital Medicine 2021;16:417-423. (C) 2021 Society of Hospital Medicine

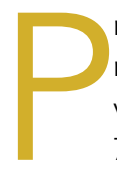

roton pump inhibitors (PPIs) are among the most commonly used drugs worldwide to treat dyspepsia and prevent gastrointestinal bleeding (GIB). ${ }^{1}$ Between $40 \%$ and $70 \%$ of hospitalized patients receive acid-suppressive therapy (AST; defined as PPIs or histamine-receptor antagonists), and nearly half of these are initiated during the inpatient stay. ${ }^{2,3}$ While up to $50 \%$ of inpatients who received a new AST were discharged on these medications, ${ }^{2}$ there were no evidence-based indications for a majority of the prescriptions. ${ }^{2,3}$

Growing evidence shows that PPIs are overutilized and may be associated with wide-ranging adverse events, such as acute and chronic kidney disease, ${ }^{4}$ Clostridium difficile infection, ${ }^{5}$ hypomagnesemia, ${ }^{6}$ and fractures. ${ }^{7}$ Because of the widespread overuse and the potential harm associated with PPIs, a concerted effort to promote their appropriate use in the inpatient setting is necessary. It is important to note that reducing the use of PPIs does not increase the risks of GIB or worsening dyspepsia. Rather, reducing overuse of PPIs lowers the risk of harm to patients. The efforts to reduce overuse, however, are complex and difficult.

This article summarizes evidence regarding interventions to reduce overuse and offers an implementation guide based

*Corresponding Author: Karen Clarke, MD, MSc, MPH; Email: summarizephilosophy@yahoo.com; Telephone: 678-857-9924.

Published online first June 16, 2021

Received: February 27, 2020; Revised: April 10, 2021; Accepted: April 13, 2021

(c) 2021 Society of Hospital Medicine DOI 10.12788/jhm.3637 on this evidence. This guide promotes value-based quality improvement and provides a blueprint for implementing an institution-wide program to reduce PPI overuse in the inpatient setting. We begin with a discussion about quality initiatives to reduce PPI overuse, followed by a review of the safety outcomes associated with reduced use of PPIs.

\section{METHODS}

A focused search of the US National Library of Medicine's PubMed database was performed to identify Englishlanguage articles published between 2000 and 2018 that addressed strategies to reduce PPI overuse for stress ulcer prophylaxis (SUP) and nonvariceal GIB. The following search terms were used: PPI and inappropriate use; acid-suppressive therapy and inappropriate use; PPI and discontinuation; acid-suppressive (or suppressant) therapy and discontinuation; SUP and cost; and histamine receptor antagonist and PPI. Inpatient or outpatient studies of patients aged 18 years or older were considered for inclusion in this narrative review, and all study types were included. The primary exclusion criterion was patients aged younger than 18 years. A manual review of the full text of the retrieved articles was performed and references were reviewed for missed citations.

\section{RESULTS}

We identified a total of 1,497 unique citations through our initial search. After performing a manual review, we excluded 1,483 of the references and added an additional 2, resulting in 16 articles 
TABLE 1. Studies Evaluating the Implementation of Institutional Guidelines and Electronic Health Records to Reduce PPI Overuse in the Hospital Setting

\begin{tabular}{lll}
\hline Study description & Intervention & Results \\
\hline Coursol and Sanzari, ${ }^{8} 2005$ & Institutional guideline & The proportion of inappropriate prophylaxis decreased from $95.7 \%$ before \\
Design: Prospective, simple pre-post study (no control group) & the intervention to $88.2 \%$ post intervention $(P=.033)$.
\end{tabular}

Preintervention period: 3 months $(n=303)$

Postintervention period: 3 months ( $n=252$ )

Setting: ICU at a regional health center in Montréal, Quebec, Canada

Herzig et al, ${ }^{11} 2015$

EHR clinical decision support

Inappropriate acid-suppressive exposure decreased from $4.0 \%$ before the intervention to $0.6 \%$ post intervention (East Campus) and from $7.7 \%$ be-

Design: Quasi-experimental pre-post study using an interrupted time series analysis fore the intervention to $2.2 \%$ post intervention (West Campus) $(P<.001)$.

Preintervention period: 4 months ( $n=14,924)$

Postintervention period: 6 months $(n=11,476)$

Setting: Academic medical center in Boston, Massachusetts

Michal et $\mathrm{al}_{,}^{10} 2016$

Design: Concurrent, simple pre-post study (no control group)

Pharmacist-led institutiona protocol

PPI discontinuation during the hospitalization increased from $41.1 \%$ before the intervention to $66 \%$ post intervention $(P=.001)$.

Preintervention period: 1 month ( $n=95)$

Postintervention period: 1 month 1 year later $(n=94)$

Setting: Community hospital medical unit in Gastonia, North Carolina

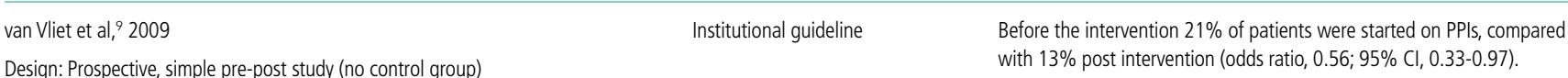

Preintervention period: Consecutively admitted patients in a

6-month period $(n=300)$

Postintervention period: Consecutively admitted patients in a 7-month period $(n=300)$

Setting: two pulmonary medicine units in Rotterdam, The Netherlands

Abbreviations: EHR, electronic health record; ICU, intensive care unit; PPI, proton pump inhibitor.

selected for inclusion. The selected articles addressed interventions falling into three main groupings: implementation of institutional guidelines with or without electronic health record (EHR)-based decision support, educational interventions alone, and multifaceted interventions. Each of these interventions is discussed in the sections that follow. Table 1, Table 2, and Table 3 summarize the results of the studies included in our narrative review.

\section{QUALITY INITIATIVES TO REDUCE PPI OVERUSE}

\section{Institutional Guidelines With or Without EHR-Based} Decision Support

Table 1 summarizes institutional guidelines, with or without EHR-based decision support, to reduce inappropriate PPI use. The implementation of institutional guidelines for the appropriate reduction of PPI use has had some success. Coursol and Sanzari evaluated the impact of a treatment algorithm on the appropriateness of prescriptions for SUP in the intensive care unit (ICU). ${ }^{8}$ Risk factors of patients in this study included mechanical ventilation for 48 hours, coagulopathy for 24 hours, postoperative transplant, severe burns, active gastrointestinal (GI) disease, multiple trauma, multiple organ failure, and septicemia. The three treatment options chosen for the algorithm were intravenous (IV) famotidine (if the oral route was unavailable or impractical), omeprazole tablets (if oral access was available), and omeprazole suspension (in cases of dysphagia and presence of nasogastric or orogastric tube). After implementation of the treatment algorithm, the proportion of inappropriate prophylaxis decreased from $95.7 \%$ to 88.2\% ( $P=.033)$, and the cost per patient decreased from $\$ 11.11$ to $\$ 8.49$ Canadian dollars $(P=.003)$.

Van Vliet et al implemented a clinical practice guideline listing specific criteria for prescribing a PPI. ${ }^{9}$ Their criteria included the presence of gastric or duodenal ulcer and use of a nonsteroidal anti-inflammatory drug (NSAID) or aspirin, plus at least one additional risk factor (eg, history of gastroduodenal hemorrhage or age $>70$ years). The proportion of patients started on PPIs during hospitalization decreased from $21 \%$ to $13 \%$ (odds ratio, 0.56; 95\% Cl, 0.33-0.97).

Michal et al utilized an institutional pharmacist-driven protocol that stipulated criteria for appropriate PPI use (eg, upper GIB, mechanical ventilation, peptic ulcer disease, gastroesophageal reflux disease, coagulopathy). ${ }^{10}$ Pharmacists in the study evaluated patients for PPI appropriateness and recommended changes in medication or discontinuation of use. This institutional intervention decreased PPI use in non-ICU hospitalized adults. Discontinuation of PPls increased from $41 \%$ of patients in the preintervention group to $66 \%$ of patients in the postintervention group $(P=.001)$. 
In addition to implementing guidelines and intervention strategies, institutions have also adopted changes to the EHR to reduce inappropriate PPI use. Herzig et al utilized a computerized clinical decision support intervention to decrease SUP in non-ICU hospitalized patients. ${ }^{11}$ Of the available response options for acid-suppressive medication, when SUP was chosen as the only indication for PPI use a prompt alerted the clinician that "[SUP] is not recommended for patients outside the [ICU]"; the alert resulted in a significant reduction in AST for the sole purpose of SUP. With this intervention, the percentage of patients who had any inappropriate acidsuppressive exposure decreased from $4.0 \%$ to $0.6 \%(P<.001)$.

\section{EDUCATION}

Table 2 summarizes educational interventions to reduce inappropriate PPI use.

Agee et al employed a pharmacist-led educational seminar that described SUP indications, risks, and costs. ${ }^{12}$ Inappropriate SUP prescriptions decreased from $55.5 \%$ to $30.5 \%$ after the intervention $(P<.0001)$. However, there was no reduction in the percentage of patients discharged on inappropriate AST.

Chui et al performed an intervention with academic detailing wherein a one-on-one visit with a physician took place, providing education to improve physician prescribing behavior. ${ }^{13}$ In this study, academic detailing focused on the most common instances for which PPIs were inappropriately utilized at that hospital (eg, surgical prophylaxis, anemia). Inappropriate use of double-dose PPIs was also targeted. Despite these efforts, no significant difference in inappropriate PPI prescribing was observed post intervention.

Hamzat et al implemented an educational strategy to reduce inappropriate PPI prescribing during hospital stays, which included dissemination of fliers, posters, emails, and presentations over a 4-week period..$^{14}$ Educational efforts targeted clinical pharmacists, nurses, physicians, and patients. Appropriate indications for PPI use in this study included peptic ulcer disease (current or previous), H pylori infection, and treatment or prevention of an NSAID-induced ulcer. The primary outcome was a reduction in PPI dose or discontinuation of PPI during the hospital admission, which increased from $9 \%$ in the preintervention (pre-education) phase to $43 \%$ during the intervention (education) phase and to $46 \%$ in the postintervention (posteducation) phase $(P=.006)$.

Liberman and Whelan also implemented an educational intervention among internal medicine residents to reduce inappropriate use of SUP; this intervention was based on practice-based learning and improvement methodology. ${ }^{15}$ They noted that the rate of inappropriate prophylaxis with AST decreased from $59 \%$ preintervention to $33 \%$ post intervention $(P<.007)$.

\section{MULTIFACETED APPROACHES}

Table 3 summarizes several multifaceted approaches aimed at reducing inappropriate PPI use. Belfield et al utilized an intervention consisting of an institutional guideline review, education, and monitoring of AST by clinical pharmacists to reduce inappropriate use of PPI for SUP. ${ }^{16}$ With this intervention, the primary outcome of total inappropriate days of AST during hospitalization decreased from 279 to 116 (48\% relative reduction in risk, $P<.01$, across 142 patients studied). Furthermore, inappropriate AST prescriptions at discharge decreased from $32 \%$ to $8 \%(P=.006)$. The one case of GIB noted in this study occurred in the control group.

Del Giorno et al combined audit and feedback with education to reduce new PPI prescriptions at the time of discharge from the hospital. ${ }^{17}$ The educational component of this intervention included guidance regarding potentially inappropriate PPI use and associated side effects and targeted multiple departments in the hospital. This intervention led to a sustained reduction in new PPI prescriptions at discharge during the 3-year study period. The annual rate of new PPI prescriptions was $19 \%, 19 \%, 18 \%$, and $16 \%$ in years $2014,2015,2016$, and 2017, respectively, in the internal medicine department (postintervention group), compared with rates of $30 \%, 29 \%$, $36 \%, 36 \%(P<.001)$ for the same years in the surgery department (control group).

Education and the use of medication reconciliation forms on admission and discharge were utilized by Gupta et al to reduce inappropriate AST in hospitalized patients from $51 \%$ prior to intervention to $22 \%$ post intervention $(P<.001) .{ }^{18}$ Furthermore, the proportion of patients discharged on inappropriate AST decreased from $69 \%$ to $20 \%(P<.001)$.

Hatch et al also used educational resources and pharmacist-led medication reconciliation to reduce use of SUP. ${ }^{19} \mathrm{Be}-$ fore the intervention, $24.4 \%$ of patients were continued on SUP after hospital discharge in the absence of a clear indication for use; post intervention, $11 \%$ of patients were continued on SUP after hospital discharge (of these patients, 8.7\% had no clear indication for use). This represented a $64.4 \%$ decrease in inappropriately prescribed SUP after discharge $(P<.0001)$.

Khalili et al combined an educational intervention with an institutional guideline in an infectious disease ward to reduce inappropriate use of SUP. ${ }^{20}$ This intervention reduced the inappropriate use of AST from $80.9 \%$ before the intervention to $47.1 \%$ post intervention $(P<.001)$.

Masood et al implemented two interventions wherein pharmacists reviewed SUP indications for each patient during daily team rounds, and ICU residents and fellows received education about indications for SUP and the implemented initiative on a bimonthly basis. ${ }^{21}$ Inappropriate AST decreased from 26.75 to 7.14 prescriptions per 100 patient-days of care $(P<.001)$.

McDonald et al combined education with a web-based quality improvement tool to reduce inappropriate exit prescriptions for PPIs. ${ }^{22}$ The proportion of PPIs discontinued at hospital discharge increased from $7.7 \%$ per month to $18.5 \%$ per month $(P=.03)$.

Finally, the initiative implemented by Tasaka et al to reduce overutilization of SUP included an institutional guideline, a 
TABLE 2. Studies Evaluating the Implementation of Education Interventions to Reduce PPI Use in the Hospital Setting

\begin{tabular}{lll}
\hline Study description & Intervention & Results \\
\hline Agee et al, ${ }^{12} 2015$ & Education & Inappropriate SUP decreased from $55.5 \%$ before the intervention to $30.5 \%$ \\
Design: Retrospective, simple pre-post study (no control group) & & post intervention $(P<.0001)$.
\end{tabular}

Preintervention period: 3 months $(n=220)$

Postintervention period: 4 months ( $n=193$ )

Setting: Family medicine residency team at an academic hospital in Fayetteville, Arkansas

\section{Chui et al, ${ }^{13} 2011$}

Design: Retrospective, simple pre-post study (no control group)

Preintervention period: First 100 patients who were prescribed a PPI during a

2-month preintervention period

Postintervention period: 100 patients who were prescribed

a PPI post intervention

Setting: Community hospital in Burnaby, British Columbia, Canada

Hamzat et $\mathrm{al}_{,}{ }^{14} 2012$

Design: Prospective, simple pre-post study (no control group)

Intervention period: Consecutively admitted older patients $(n=440)$

Setting: Teaching hospital in Aberdeen, Scotland

Liberman and Whelan, ${ }^{15} 2006$

Design: Prospective, simple pre-post cohort study (no control group)

Preintervention period: Consecutively admitted patients $(n=99)$

Postintervention period: 1-month and 6-month postintervention periods,

consecutively admitted patients ( $n=102$ and $n=95$, respectively)

Setting: Academic medical center in Chicago, Illinois

Education

Education

Abbreviations: PPI, proton pump inhibitor; SUP, stress ulcer prophylaxis. pharmacist-led intervention, and an institutional education and awareness campaign. ${ }^{23}$ Their initiative led to a reduction in inappropriate SUP both at the time of transfer out of the ICU (8\% before intervention, $4 \%$ post intervention, $P=.54$ ) and at the time of discharge from the hospital $7 \%$ before intervention, $0 \%$ post intervention, $P=.22$ ).

\section{REDUCING PPI USE AND SAFETY OUTCOMES}

Proton pump inhibitors are often initiated in the hospital setting, with up to half of these new prescriptions continued at discharge. ${ }^{2,24,25}$ Inappropriate prescriptions for PPIs expose patients to excess risk of long-term adverse events. ${ }^{26}$ Deescalating PPIs, however, raises concern among clinicians and patients for potential recurrence of dyspepsia and GIB. There is limited evidence regarding long-term safety outcomes (including GIB) following the discontinuation of PPIs deemed to have been inappropriately initiated in the hospital. In view of this, clinicians should educate and monitor individual patients for symptom relapse to ensure timely and appropriate resumption of AST.

\section{LIMITATIONS}

Our literature search for this narrative review and implementation guide has limitations. First, the time frame we included (2000-2018) may have excluded relevant articles published before our starting year. We did not include articles published before 2000 based on concerns these might contain outdated information. Also, there may have been incomplete retrieval of relevant studies/articles due to the labor-intensive nature involved in determining whether PPI prescriptions are appropriate or inappropriate.

We noted that interventional studies aimed at reducing overuse of PPIs were often limited by a low number of participants; these studies were also more likely to be single-center interventions, which limits generalizability. In addition, the studies often had low methodological rigor and lacked randomization or controls. Moreover, to fully evaluate the sustainability of interventions, some of the studies had a limited postimplementation period. For multifaceted interventions, the efficacy of individual components of the interventions was not clearly evaluated. Moreover, there was a high risk of bias in many of the included studies. Some of the larger studies used overall AST prescriptions as a surrogate for more appropriate use. It would be advantageous for a site to perform a pilot study that provides well-defined parameters for appropriate prescribing, and then correlate with the total number of prescriptions (automated and much easier) thereafter. Further, although the evidence regarding 
TABLE 3. Studies Evaluating the Implementation of a Multifaceted Approach to Reduce PPI Overuse in the Hospital Setting

\begin{tabular}{lll}
\hline Study description & Intervention & Results \\
\hline Belfield et $a{ }^{16}{ }^{16} 2017$ & Institutional guideline & There was a $31 \%$ absolute reduction in inappropriate AST patient-days \\
Design: Retrospective, simple pre-post study (no control group) & Education & $(P<.01)$ and a 24\% absolute reduction in patients discharged on AST \\
Preintervention period: 1 month $(\mathrm{n}=300)$ & Pharmacist-led audit and feedback & $(P<.006)$.
\end{tabular}

Postintervention period: 1 month $(n=508)$

Setting: Adult non-ICU patients admitted to the hospitalist service at an academic medical center

Del Giorno et al, ${ }^{17} 2018$

Design: Multicenter longitudinal quasi-experimental, simple pre-post study (nonequivalent control group)

Intervention period: 36 months (44,973 admissions)

Setting: 5 public teaching hospitals in the Italian-speaking region of Switzerland; internal medicine department intervention group compared to surgery department

Gupta et al, $^{18} 2013$

Design: Retrospective, simple pre-post study (no control group)

Preintervention period: 3 months $(n=400)$

Postintervention period: 3 months $(n=270)$

Setting: General medical service at a university hospital in Jacksonville, Florida

Hatch et al, ${ }^{19} 2010$

Design: Retrospective, simple pre-post study (no control group)

Intervention period: Consecutively admitted adult patients $(n=356)$

Setting: ICU at a teaching hospital in Madison, Wisconsin

Khalili et al, ${ }^{20} 2010$

Design: Prospective, simple pre-post study (no control group)

Preintervention period: 4 months $(n=262)$

Postintervention period: 4 months ( $n=240)$

Setting: Infectious diseases ward of academic medical center in Tehran, Iran

Masood et al, ${ }^{21} 2018$

Design: Retrospective, observational, simple pre-post study (no control group)

Preintervention period: 1 month, all medical patients admitted to the ICU

Postintervention period: 1 month, all medical patients admitted to the ICU

Setting: ICU in academic medical center in Syracuse, New York

McDonald et al, ${ }^{22} 2015$

Design: Prospective, simple pre-post study (no control group)

Preintervention period: Consecutively admitted patients in a 5-month

period $(n=464)$

Postintervention period: Consecutively admitted patients in a 5.5-month period

$(n=640)$

Setting: Academic medical center in Montreal, Quebec, Canada

Tasaka et al, ${ }^{23} 2014$

Design: Retrospective, observational cohort, simple pre-post study

(no control group)

Preintervention period: Pre-computerized provider order entry $(n=54)$ and post-

computerized order entry $(n=75)$
Education

Pharmacists discussed appropriateness of SUP with prescribers
The annual rate of new PPI prescriptions for internal medicine showed a decreasing trend: $19 \%, 19 \%, 18 \%$, and $16 \%$ in years $2014,2015,2016$, and 2017, respectively $(P<.001,2014$ vs 2017; $P$-for-trend $<.001)$, while an increasing rate was found in the surgery department (which had no intervention) in the same years.
Education

Required justification of any new

discharge prescriptions
AST use decreased from $70 \%$ before the intervention to $37 \%$ post intervention $(P<.001)$. Before the intervention, the rate of inappropriate AST prescriptions was $51 \%$, compared with $22 \%$ post intervention $(P<.02)$.
Postintervention period: Post-bundled intervention $(n=56)$

Setting: Medical and surgical ICUs at academic medical center in San Francisco,

California
Pharmacist-led institutional protocol AST use declined from $80.9 \%$ of patients before the intervention to $47.1 \%$ Education
Inappropriate continuation of gastric acid suppressants after hospital discharge decreased from $24.4 \%$ before the intervention to $8.7 \%$ post intervention $(P<.0001)$
Pharmacist rounding with medical team provided guidance about SUP use

Education
The incidence of inappropriate SUP was 26.75 per 100 patient-days before the intervention compared with 7.14 per 100 patient-days post intervention $(P<.001)$.

Abbreviations: AST, acid-suppressive therapy; EHR, electronic health record; ICUs, intensive care units; PPI, proton pump inhibitor. 
appropriate PPI use for SUP and GIB has shifted rapidly in recent years, society guidelines have not been updated to reflect this change. As such, quality improvement interventions have predominantly focused on reducing PPI use for the indications reflected by these guidelines.

\section{IMPLEMENTATION BLUEPRINT}

The following are our recommendations for successfully implementing an evidence-based, institution-wide initiative to promote the appropriate use of PPIs during hospitalization. These recommendations are informed by the evidence review and reflect the consensus of the combined committees coauthoring this review.

For an initiative to succeed, participation from multiple disciplines is necessary to formulate local guidelines and design and implement interventions. Such an interdisciplinary approach requires advocates to closely monitor and evaluate the program; sustainability will be greatly facilitated by the active engagement of key stakeholders, including the hospital's executive administration, supply chain, pharmacists, and gastroenterologists. Lack of adequate buy-in on the part of key stakeholders is a barrier to the success of any intervention. Accordingly, before selecting a particular intervention, it is important to understand local factors driving the overuse of PPI.

\section{Develop evidence-based institutional guidelines for both} SUP and nonvariceal upper GIB through an interdisciplinary workgroup.

- Establish an interdisciplinary group including, but not limited to, pharmacists, hospitalists, gastroenterologists, and intensivists so that changes in practice will be widely adopted as institutional policy.

- Incorporate the best evidence and clearly convey appropriate and inappropriate uses.

\section{Integrate changes to the EHR.}

- If possible, the EHR should be leveraged to implement changes in PPI ordering practices.

- While integrating changes to the EHR, it is important to consider informatics and implementation science, since the utility of hard stops and best practice alerts has been questioned in the setting of operational inefficiencies and alert fatigue.

- Options for integrating changes to the EHR include the following:

- Create an ordering pathway that provides clinical decision support for PPI use.

- Incorporate a best practice alert in the EMR to notify clinicians of institutional guidelines when they initiate an order for PPI outside of the pathway.

- Consider restricting the authority to order IV PPIs by requiring a code or password or implement another means of using the EHR to limit the supply of PPI.

- Limit the duration of IV PPI by requiring daily renewal of IV PPI dosing or by altering the period of time that use of IV PPI is permitted (eg, 48 to 72 hours).
- PPIs should be removed from any current order sets that include medications for SUP.

3. Foster pharmacy-driven interventions.

- Consider requiring pharmacist approval for IV PPIs.

- Pharmacist-led review and feedback to clinicians for discontinuation of inappropriate PPIs can be effective in decreasing inappropriate utilization.

4. Provide education, audit data, and obtain feedback.

- Data auditing is needed to measure the efficacy of interventions. Outcome measures may include the number of non-ICU and ICU patients who are started on a PPI during an admission; the audit should be continued through discharge. A process measure may be the number of pharmacist calls for inappropriate PPIs. A balancing measure would be ulcer-specific upper GIB in patients who do not receive SUP during their admission. (Upper GIB from other etiologies, such as varices, portal hypertensive gastropathy, and Mallory-Weiss tear would not be affected by PPI SUP.)

- Run or control charts should be utilized, and data should be shared with project champions and ordering clinicians-in real time if possible.

- Project champions should provide feedback to colleagues; they should also work with hospital leadership to develop new strategies to improve adherence.

- Provide ongoing education about appropriate indications for PPIs and potential adverse effects associated with their use. Whenever possible, point-of-care or just-in-time teaching is the preferred format.

\section{CONCLUSION}

Excessive use of PPls during hospitalization is prevalent; however, quality improvement interventions can be effective in achieving sustainable reductions in overuse. There is a need for the American College of Gastroenterology to revisit and update their guidelines for management of patients with ulcer bleeding to include stronger evidence-based recommendations on the proper use of PPIs. ${ }^{27}$ These updated guidelines could be used to update the implementation blueprint.

Quality improvement teams have an opportunity to use the principles of value-based healthcare to reduce inappropriate PPI use. By following the blueprint outlined in this article, institutions can safely and effectively tailor the use of PPIs to suitable patients in the appropriate settings. Reduction of PPI overuse can be employed as an institutional catalyst to promote implementation of further value-based measures to improve efficiency and quality of patient care.

Disclosures: The authors report no conflicts of interest.

The contributing authors represent a joint collaboration between the High Value Practice Academic Alliance and Society of Hospital Medicine's High Value Care Committee. 


\section{References}

1. Savarino $V$, Marabotto $E$, Zentilin $P$, et al. Proton pump inhibitors: use and misuse in the clinical setting. Exp Rev Clin Pharmacol. 2018;11(11):11231134. https://doi.org/10.1080/17512433.2018.1531703

2. Nardino RJ, Vender RJ, Herbert PN. Overuse of acid-suppressive therapy in hospitalized patients. Am J Gastroenterol. 2000;95(11):3118-3122. https:// doi.org/10.1111/j.1572-0241.2000.03259.x

3. Ahrens D, Behrens G, Himmel W, Kochen MM, Chenot JF. Appropriateness of proton pump inhibitor recommendations at hospital discharge and continuation in primary care. Int J Clin Pract. 2012;66(8):767-773. https://doi. org/10.1111/j.1742-1241.2012.02973.x

4. Moledina DG, Perazella MA. PPIs and kidney disease: from AIN to CKD. J Nephrol. 2016;29(5):611-616. https://doi.org/10.1007/s40620-016-0309-2

5. Kwok CS, Arthur AK, Anibueze Cl, Singh S, Cavallazzi R, Loke YK. Risk of Clostridium difficile infection with acid suppressing drugs and antibiotics: meta-analysis. Am J Gastroenterol. 2012;107(7):1011-1019. https://doi. org/10.1038/ajg.2012.108

6. Cheungpasitporn W, Thongprayoon C, Kittanamongkolchai W, et al. Proton pump inhibitors linked to hypomagnesemia: a systematic review and meta-analysis of observational studies. Ren Fail. 2015;37(7):1237-1241. https://doi.org/10.3109/0886022x.2015.1057800

7. Yang YX, Lewis JD, Epstein S, Metz DC. Long-term proton pump inhibitor therapy and risk of hip fracture. JAMA. 2006;296(24):2947-2953. https://doi. org/10.1001/jama.296.24.2947

8. Coursol CJ, Sanzari SE. Impact of stress ulcer prophylaxis algorithm study. Ann Pharmacother. 2005;39(5):810-816. https://doi.org/10.1345/aph.1d129

9. van Vliet EPM, Steyerberg EW, Otten HJ, et al. The effects of guideline implementation for proton pump inhibitor prescription on two pulmonary medicine wards. Aliment Pharmacol Ther. 2009;29(2):213-221. https://doi. org/10.1111/j.1365-2036.2008.03875.x

10. Michal J, Henry T, Street C. Impact of a pharmacist-driven protocol to decrease proton pump inhibitor use in non-intensive care hospitalized adults. Am J Health Syst Pharm. 2016;73(17 Suppl 4):S126-S132. https:// doi.org/10.2146/ajhp150519

11. Herzig SJ, Guess JR, Feinbloom DB, et al. Improving appropriateness of acid-suppressive medication use via computerized clinical decision support. J Hosp Med. 2015;10(1):41-45. https://doi.org/10.1002/jhm.2260

12. Agee C, Coulter L, Hudson J. Effects of pharmacy resident led education on resident physician prescribing habits associated with stress ulcer prophylaxis in non-intensive care unit patients. Am J Health Syst Pharm 2015;72(11 Suppl 1):S48-S52. https://doi.org/10.2146/sp150013

13. Chui D, Young F, Tejani AM, Dillon EC. Impact of academic detailing on proton pump inhibitor prescribing behaviour in a community hospital. Can Pharm J (Ott). 2011;144(2):66-71. https://doi.org/10.3821/1913$701 X-144.2 .66$

14. Hamzat H, Sun H, Ford JC, Macleod J, Soiza RL, Mangoni AA. Inappropriate prescribing of proton pump inhibitors in older patients: effects of an educational strategy. Drugs Aging. 2012;29(8):681-690. https://doi. org/10.1007/bf03262283

15. Liberman JD, Whelan CT. Brief report: Reducing inappropriate usage of stress ulcer prophylaxis among internal medicine residents. A practice-based educational intervention. J Gen Intern Med. 2006;21(5):498-500. https://doi.org/10.1111/j.1525-1497.2006.00435.x

16. Belfield KD, Kuyumjian AG, Teran R, Amadi M, Blatt M, Bicking K. Impact of a collaborative strategy to reduce the inappropriate use of acid suppressive therapy in non-intensive care unit patients. Ann Pharmacother 2017;51(7):577-583. https://doi.org/10.1177/1060028017698797

17. Del Giorno R, Ceschi A, Pironi M, Zasa A, Greco A, Gabutti L. Multifaceted intervention to curb in-hospital over-prescription of proton pump inhibitors: a longitudinal multicenter quasi-experimental before-and-after study. Eur J Intern Med. 2018;50:52-59. https://doi.org/10.1016/j.ejim.2017.11.002

18. Gupta R, Marshall J, Munoz JC, Kottoor R, Jamal MM, Vega KJ. Decreased acid suppression therapy overuse after education and medication reconciliation. Int J Clin Pract. 2013;67(1):60-65. https://doi.org/10.1111/ijcp.12046

19. Hatch JB, Schulz L, Fish JT. Stress ulcer prophylaxis: reducing non-indicated prescribing after hospital discharge. Ann Pharmacother. 2010;44(10):15651571. https://doi.org/10.1345/aph.1p167

20. Khalili H, Dashti-Khavidaki S, Hossein Talasaz AH, Tabeefar H, Hendoiee N. Descriptive analysis of a clinical pharmacy intervention to improve the appropriate use of stress ulcer prophylaxis in a hospital infectious disease ward. J Manag Care Pharm. 2010;16(2):114-121. https://doi.org/10.18553/ jmcp.2010.16.2.114

21. Masood U, Sharma A, Bhatti Z, et al. A successful pharmacist-based quality initiative to reduce inappropriate stress ulcer prophylaxis use in an academic medical intensive care unit. Inquiry. 2018;55:46958018759116. https://doi.org/10.1177/0046958018759116

22. McDonald EG, Jones J, Green L, Jayaraman D, Lee TC. Reduction of inappropriate exit prescriptions for proton pump inhibitors: a before-after study using education paired with a web-based quality-improvement tool. J Hosp Med. 2015;10(5):281-286. https://doi.org/10.1002/jhm.2330

23. Tasaka CL, Burg C, VanOsdol SJ, et al. An interprofessional approach to reducing the overutilization of stress ulcer prophylaxis in adult medical and surgical intensive care units. Ann Pharmacother. 2014;48(4):462-469. https://doi.org/10.1177/1060028013517088

24. Zink DA, Pohlman M, Barnes M, Cannon ME. Long-term use of acid suppression started inappropriately during hospitalization. Aliment Pharmacol Ther. 2005;21(10):1203-1209. https://doi.org/10.1111/j.13652036.2005.02454.x

25. Pham CQ, Regal RE, Bostwick TR, Knauf KS. Acid suppressive therapy use on an inpatient internal medicine service. Ann Pharmacother. 2006;40 (7-8):1261-1266. https://doi.org/10.1345/aph.1g703

26. Schoenfeld AJ, Grady D. Adverse effects associated with proton pump inhibitors [editorial]. JAMA Intern Med. 2016;176(2):172-174. https://doi. org/10.1001/jamainternmed.2015.7927

27. Laine $L$, Jensen DM. Management of patients with ulcer bleeding. Am J Gastroenterol. 2012;107(3):345-360; quiz 361. https://doi.org/10.1038/ ajg.2011.480 